\title{
ITERATIVE SOLUTION OF PIECEWISE LINEAR SYSTEMS AND APPLICATIONS TO FLOWS IN POROUS MEDIA
}

\author{
LUIGI BRUGNANO* AND VINCENZO CASULLI ${ }^{\dagger}$
}

\begin{abstract}
For the efficient mathematical modelling of free-surface hydrodynamic problems, it is required the solution of special linear systems whose coefficient matrix is a piecewise constant function of the solution itself. In so doing one may fulfill relevant physical constraints. In particular, the existence, the uniqueness, and two constructive iterative methods to solve a piecewise linear system of the form

$$
\max [\boldsymbol{l}, \min (\boldsymbol{u}, \mathbf{x})]+T \mathbf{x}=\mathbf{b},
$$

are analysed. The methods are shown to have a finite termination property, i.e., they converge to an exact solution in a finite number of steps and, actually, they converge very quickly, as confirmed by a few numerical tests, which are derived from the mathematical modelling of flows in porous media.
\end{abstract}

Key words. piecewise linear systems, free-surface hydrodynamics, wetting and drying, flows in porous media, confined-unconfined aquifers.

AMS subject classifications. 90C33, 90C53, 90C06, 76M20, 76S05.

1. Introduction. The problem which is here analysed is that of finding a solution to

$$
\max [\boldsymbol{l}, \min (\boldsymbol{u}, \mathbf{x})]+T \mathbf{x}=\mathbf{b},
$$

where the max and min functions are applied componentwise, $\boldsymbol{l}, \boldsymbol{u}, \mathbf{b} \in \mathbb{R}^{n}$ are known vectors, $\boldsymbol{l}=\left(l_{i}\right) \leq \boldsymbol{u}=\left(u_{i}\right)$, and $T$ is a symmetric and (at least) positive semidefinite matrix of dimension $n$ satisfying either one of the following properties

T1 : $T$ is a Stieltjes matrix, i.e., a symmetric $M$-matrix (then non-singular, see, e.g., $[10])$; or

T2 : $\operatorname{null}(T) \equiv \operatorname{span}(\mathbf{v})$ with $\mathbf{v}>\mathbf{0}$, and $T+D$ satisfies $\mathbf{T 1}$ for all diagonal matrices $D \gtreqless O$ (i.e., $D \geq O$ and $D \neq O$ ).

By denoting with $I$ the identity matrix, if $\mathbf{x}$ is a solution of the linear system $(I+T) \mathbf{x}=\mathbf{b}$, and $\boldsymbol{l} \leq \mathbf{x} \leq \boldsymbol{u}$, then $\mathbf{x}$ is the unique solution of (1.1) and can be efficiently determined by a preconditioned conjugate gradient method.

If $\mathbf{x}$ is a solution of the problem $\max (\boldsymbol{l}, \mathbf{x})+T \mathbf{x}=\mathbf{b}$, and $\mathbf{x} \leq \boldsymbol{u}$, then $\mathbf{x}$ is a solution of (1.1). Similarly, if $\mathbf{x}$ is a solution of the problem $\min (\boldsymbol{u}, \mathbf{x})+T \mathbf{x}=\mathbf{b}$, and $\mathbf{x} \geq \boldsymbol{l}$, then $\mathbf{x}$ is a solution of (1.1). In both these cases $\mathbf{x}$ can be conveniently determined with a simple Newton-type method as described in Reference [3].

In general, System (1.1) can be written, equivalently, as

$$
P(\mathbf{x})(\mathbf{x}-\boldsymbol{l})-Q(\mathbf{x})(\mathbf{x}-\boldsymbol{u})+T \mathbf{x}=\mathbf{b}-\boldsymbol{l},
$$

where $P(\mathbf{x})$ e $Q(\mathbf{x})$ are diagonal matrices whose diagonal entries are step functions given by

$$
p\left(x_{i}\right)=\left\{\begin{array}{ll}
1 & \text { if } x_{i} \geq l_{i}, \\
0 & \text { otherwise, }
\end{array} \quad q\left(x_{i}\right)= \begin{cases}1 & \text { if } x_{i}>u_{i} \\
0 & \text { otherwise }\end{cases}\right.
$$

*Dipartimento di Matematica "U. Dini", Viale Morgagni 67/A, 50134 Firenze (Italy), e-mail: luigi.brugnano@unifi.it

${ }^{\dagger}$ Dipartimento di Ingegneria Civile e Ambientale, Via Mesiano 77, 38050 Trento (Italy), e-mail: vincenzo.casulli@unitn.it 
Because of the characterization (1.3) of System (1.2), this will be said to be a piecewise linear system (see also [3]).

The following easy properties are here stated, for later reference.

LEMma 1. With reference to (1.2)-(1.3), $\forall \mathbf{x} \in \mathbb{R}^{n}$ one has

- $I \geq P(\mathbf{x}) \geq Q(\mathbf{x}) \geq O$;

- for any diagonal matrix $R$ with binary diagonal entries (0 or 1 ),

$$
[P(\mathbf{x})-R](\mathbf{x}-\mathbf{l}) \geq \mathbf{0}, \quad[Q(\mathbf{x})-R](\mathbf{x}-\boldsymbol{u}) \geq \mathbf{0} .
$$

Problems in the form (1.1) arise in the numerical solution of free-surface hydrodynamic problems, so that their efficient solution is of interest in applications (see, e.g. $[7,8,12,13]$; see also [5]).

By suitably increasing its dimensionality, problem (1.1) could be written as a nonsmooth system of equations [14]. In particular, it can be reformulated as a box constrained semismooth problem, whose numerical solution is an active field of investigation (see, e.g., $[2,11]$ and the references contained therein). Alternatively, if the dimension of the problem is left unchanged, System (1.2) could be solved by means of some kind of Picard iteration. Nevertheless, for all such methods, convergence to the solution, in general, occurs only in the limit of an infinite number of iterations.

Moreover, a straightforward application of the Newton method could even lead to undefined and non-converging iterations. Consider, for example, problem (1.2) with

$$
\boldsymbol{l}=\left(\begin{array}{l}
0 \\
0
\end{array}\right), \quad \boldsymbol{u}=\left(\begin{array}{l}
1 \\
1
\end{array}\right), \quad \mathbf{b}=\left(\begin{array}{r}
-3 \\
4
\end{array}\right), \quad T=\left(\begin{array}{rr}
1 & -1 \\
-1 & 1
\end{array}\right),
$$

and the following Newton-type iteration for its solution

$$
\begin{aligned}
& P^{0}=I, \quad Q^{0}=O, \\
& {\left[P^{k-1}-Q^{k-1}+T\right] \mathbf{x}^{k}=\mathbf{b}-\left(I-P^{k-1}\right) \boldsymbol{l}-Q^{k-1} \boldsymbol{u}, \quad k=1,2, \ldots,}
\end{aligned}
$$

where, hereafter, $P^{k}=P\left(\mathbf{x}^{k}\right)$ and $Q^{k}=Q\left(\mathbf{x}^{k}\right)$ for all $k \geq 1$. It turns out that such an iteration becomes undefined after just one iteration. In fact,

$$
\mathrm{x}^{1}=\frac{1}{3}\left(\begin{array}{r}
-2 \\
5
\end{array}\right) \quad \Longrightarrow \quad P^{1}=Q^{1}=\left(\begin{array}{ll}
0 & \\
& 1
\end{array}\right) .
$$

Consequently, iteration (1.5) is no longer defined and $\mathbf{x}^{1}$ is clearly not a solution. On the other hand, if we consider the following modified Newton method,

$$
\begin{aligned}
\mathbf{x}^{k}= & \mathbf{x}^{k-1}-(I+T)^{-1}\left[P^{k-1}\left(\mathbf{x}^{k-1}-\boldsymbol{l}\right)-Q^{k-1}\left(\mathbf{x}^{k-1}-\boldsymbol{u}\right)\right. \\
& \left.+T \mathbf{x}^{k-1}-\mathbf{b}+\boldsymbol{l}\right], \quad k=1,2, \ldots,
\end{aligned}
$$

global convergence can be readily shown. However, starting from $\mathbf{x}^{0}=\mathbf{0}$, this problem requires 34 iterations to obtain an approximate solution within 15 digits accuracy.

In order to overcome these potential drawbacks, two simple nested (inner-outer) iterative algorithms will be defined. Often, inner-outer iterations require more computation than one-level iterations. Nevertheless, the two nested iterations here proposed are able to guarantee convergence to the exact solution in a finite number of iterations. For the above example, a total of 2 iterations are sufficient.

The existence of a solution for problem (1.2) is studied in Section 2. In Section 3 the conditions for its uniqueness are analysed. Section 4 describes some numerical examples derived from the mathematical modelling of flows in porous media. Finally, some concluding remarks are contained in Section 5. 


\section{Algorithm 1}

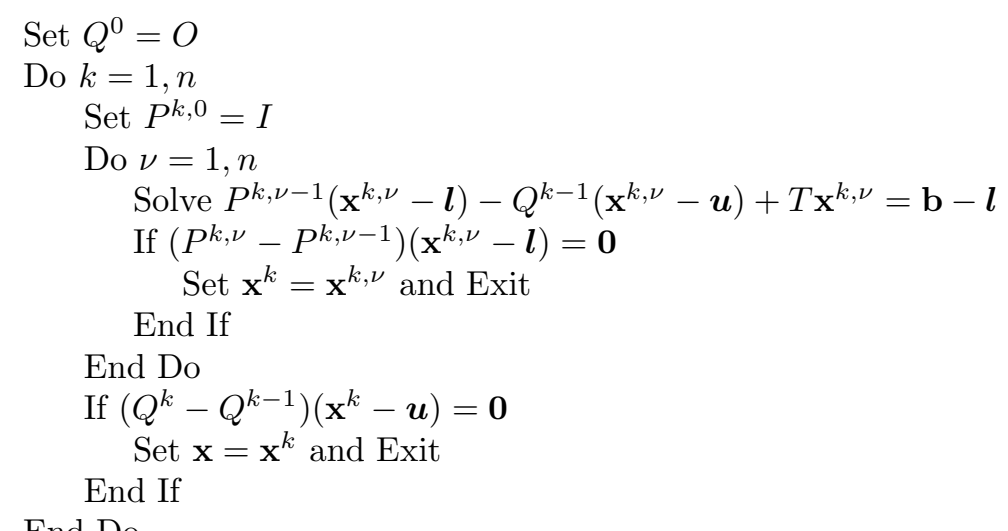

End Do

2. Existence of a solution. The existence of a solution for System (1.2) will be established constructively in this section by means of two nested iterative methods.

First, by setting $Q^{0}=O$, a sequence of iterates $\left\{\mathbf{x}^{k}\right\}$ is determined from

$$
P^{k}\left(\mathbf{x}^{k}-\boldsymbol{l}\right)-Q^{k-1}\left(\mathbf{x}^{k}-\boldsymbol{u}\right)+T \mathbf{x}^{k}=\mathbf{b}-\boldsymbol{l}, \quad k=1,2, \ldots
$$

A straightforward stopping criterion for this iteration is provided by the following result.

Lemma 2. An exact solution of (1.2) is obtained from (2.1) when, for some $k \geq 1$, one has

$$
\left(Q^{k}-Q^{k-1}\right)\left(\mathbf{x}^{k}-\boldsymbol{u}\right)=\mathbf{0} .
$$

For all $k=1,2, \ldots$, a vector $\mathbf{x}^{k}$ solving (2.1) can be obtained by inner iterations, by setting $P^{k, 0}=I$ and

$$
P^{k, \nu-1}\left(\mathbf{x}^{k, \nu}-\boldsymbol{l}\right)-Q^{k-1}\left(\mathbf{x}^{k, \nu}-\boldsymbol{u}\right)+T \mathbf{x}^{k, \nu}=\mathbf{b}-\boldsymbol{l}, \quad \nu=1,2, \ldots,
$$

where $P^{k, \nu}=P\left(\mathbf{x}^{k, \nu}\right)$ for all $k, \nu \geq 1$. A straightforward stopping criterion for the inner iterations (2.3) is provided by the following lemma.

Lemma 3. An exact solution of (2.1) is obtained from (2.3) when, for some $\nu \geq 1$, one has

$$
\left(P^{k, \nu}-P^{k, \nu-1}\right)\left(\mathbf{x}^{k, \nu}-\boldsymbol{l}\right)=\mathbf{0} .
$$

This nested iterative scheme can be summarized into Algorithm 1. As one may observe, a finite upper bound for both the inner and the outer iterations has been specified: in the sequel it will be shown that these bounds are high enough to guarantee convergence to an exact solution. The following two results prove that Algorithm 1 is well defined.

TheOrEm 1. If $T$ satisfies $\mathbf{T} 2$ and

$$
\mathbf{v}^{T} \boldsymbol{l} \leq \mathbf{v}^{T} \mathbf{b} \leq \mathbf{v}^{T} \boldsymbol{u},
$$


then for all $k$ and $\nu$, until convergence, $P^{k, \nu-1}-Q^{k-1}+T$ is Stieltjes and, consequently, Algorithm 1 is well defined.

Proof One proceeds by double induction. For $k=1$ one has $P^{1,0}=I$ and $Q^{0}=O$. Thus, $P^{1,0}-Q^{0}+T$ is Stieltjes and the first inner iterate is well defined. Then, for $\nu \geq 1$ one assumes that $P^{1, \nu-1}-Q^{0}+T$ is Stieltjes, so that $\mathbf{x}^{1, \nu}$ can be uniquely determined from (2.3) and $P^{1, \nu} \geq O$. Now, since $Q^{0}=O$, if $P^{1, \nu} \neq O$ one has that $P^{1, \nu}-Q^{0}+T$ is Stieltjes and, consequently, the subsequent $(\nu+1)$-st inner iterate is well defined. Alternatively, if $P^{1, \nu}=O$, Equation (2.3) can be written as

$$
-\left(P^{1, \nu}-P^{1, \nu-1}\right)\left(\mathbf{x}^{1, \nu}-\boldsymbol{l}\right)+T \mathbf{x}^{1, \nu}=\mathbf{b}-\boldsymbol{l} .
$$

Thus, from Lemma 1 and (2.5) one obtains

$$
0 \geq-\mathbf{v}^{T}\left(P^{1, \nu}-P^{1, \nu-1}\right)\left(\mathbf{x}^{1, \nu}-l\right)=\mathbf{v}^{T}(\mathbf{b}-\boldsymbol{l}) \geq 0,
$$

so that, $\left(P^{1, \nu}-P^{1, \nu-1}\right)\left(\mathbf{x}^{1, \nu}-\boldsymbol{l}\right)=\mathbf{0}$, which is the exit condition for the inner loop. Hence, when $k=1$, the inner iterates (2.3) are well defined for all $\nu$ until convergence.

Next, for $k>1$, one assumes that the $k$-th external cycle has been successfully completed so that $\mathbf{x}^{k}$ is a solution of (2.1) and $Q^{k} \leq I$. Now, Equation (2.1) can also be written as

$$
\left(P^{k}-Q^{k}\right)\left(\mathbf{x}^{k}-\boldsymbol{l}\right)+\left(Q^{k}-Q^{k-1}\right)\left(\mathbf{x}^{k}-\boldsymbol{u}\right)+T \mathbf{x}^{k}=\mathbf{b}-\boldsymbol{l}-Q^{k}(\boldsymbol{u}-\boldsymbol{l}),
$$

which, from Lemma 1, implies

$$
\mathbf{v}^{T}\left[\mathbf{b}-\boldsymbol{l}-Q^{k}(\boldsymbol{u}-\boldsymbol{l})\right] \geq 0 .
$$

Moreover, if $Q^{k}=I$, one has $P^{k}=I$ and (2.6) reduces to

$$
\left(Q^{k}-Q^{k-1}\right)\left(\mathbf{x}^{k}-\boldsymbol{u}\right)+T \mathbf{x}^{k}=\mathbf{b}-\boldsymbol{u} .
$$

Thus, from Lemma 1, one has

$$
0 \leq \mathbf{v}^{T}\left(Q^{k}-Q^{k-1}\right)\left(\mathbf{x}^{k}-\boldsymbol{u}\right)=\mathbf{v}^{T}(\mathbf{b}-\boldsymbol{u}) \leq 0,
$$

so that, $\left(Q^{k}-Q^{k-1}\right)\left(\mathbf{x}^{k}-\boldsymbol{u}\right)=\mathbf{0}$, which is the exit condition for the outer loop. Alternatively, if $Q^{k} \neq I$ one has $P^{k+1,0}=I \supsetneqq Q^{k}$. Hence $P^{k+1,0}-Q^{k}+T$ is Stieltjes, and the first one of the $(k+1)$-st inner iterates is well defined. Then, for $\nu \geq 1$, assuming that $P^{k+1, \nu-1}-Q^{k}+T$ is Stieltjes, the $\nu$-th inner iterate is well defined and $\mathbf{x}^{k+1, \nu}$ is uniquely determined from

$$
P^{k+1, \nu-1}\left(\mathbf{x}^{k+1, \nu}-\boldsymbol{l}\right)-Q^{k}\left(\mathbf{x}^{k+1, \nu}-\boldsymbol{u}\right)+T \mathbf{x}^{k+1, \nu}=\mathbf{b}-\boldsymbol{l}
$$

and satisfies

$$
\mathbf{x}^{k+1, \nu} \geq \mathbf{x}^{k}
$$

In fact, by equating the left-hand sides of (2.1) and (2.8), one has

$$
\left(P^{k+1, \nu-1}-Q^{k}+T\right) \mathbf{x}^{k+1, \nu}=\left(P^{k+1, \nu-1}-Q^{k}+T\right) \mathbf{x}^{k}+\boldsymbol{\xi}^{k, \nu},
$$

where, from Lemma 1,

$$
\boldsymbol{\xi}^{k, \nu}=\left(P^{k}-P^{k+1, \nu-1}\right)\left(\mathbf{x}^{k}-\boldsymbol{l}\right)+\left(Q^{k}-Q^{k-1}\right)\left(\mathbf{x}^{k}-\boldsymbol{u}\right) \geq \mathbf{0} .
$$


Hence, because $\left(P^{k+1, \nu-1}-Q^{k}+T\right)^{-1}>O$, inequality (2.9) follows and implies $P^{k+1, \nu} \geq P^{k} \geq Q^{k}$. In order to complete the proof, note that if $P^{k+1, \nu} \gtreqless Q^{k}$, then $P^{k+1, \nu}-Q^{k}+T$ is Stieltjes. Consequently, the corresponding $(\nu+1)$-st inner iterate is well defined. Alternatively, if $P^{k+1, \nu}=Q^{k}$, Equation (2.8) becomes

$$
-\left(P^{k+1, \nu}-P^{k+1, \nu-1}\right)\left(\mathbf{x}^{k+1, \nu}-\boldsymbol{l}\right)+T \mathbf{x}^{k+1, \nu}=\mathbf{b}-\boldsymbol{l}-Q^{k}(\boldsymbol{u}-\boldsymbol{l}) .
$$

Consequently, from Lemma 1 and (2.7), one has

$$
0 \geq-\mathbf{v}^{T}\left(P^{k+1, \nu}-P^{k+1, \nu-1}\right)\left(\mathbf{x}^{k+1, \nu}-\boldsymbol{l}\right)=\mathbf{v}^{T}\left[\mathbf{b}-\boldsymbol{l}-Q^{k}(\boldsymbol{u}-\boldsymbol{l})\right] \geq 0,
$$

so that, $\left(P^{k+1, \nu}-P^{k+1, \nu-1}\right)\left(\mathbf{x}^{k+1, \nu}-\boldsymbol{l}\right)=\mathbf{0}$, which is the exit condition for the inner loop. Hence, when $k>1$, the inner iterates are well defined for all $\nu$ until convergence.

Corollary 1. If T satisfies $\mathbf{T} \mathbf{1}$, then for all $k$ and $\nu$, until convergence, $P^{k, \nu-1}-$ $Q^{k-1}+T$ is Stieltjes and, consequently, Algorithm 1 is well defined.

Proof For $k=1$ one has $P^{1, \nu} \geq O=Q^{0}$, so that the corresponding inner iterations are well defined for all $\nu$, since $T$ satisfies $\mathbf{T} \mathbf{1}$.

Next, for $k>1$, by using similar arguments as those used in the proof of Theorem 1 , one obtains that $(2.9)$ holds true and, consequently, $P^{k+1, \nu-1} \geq P^{k} \geq Q^{k}$. Hence $P^{k+1, \nu-1}-Q^{k}+T$ is Stieltjes for all $k$ and for all $\nu$.

The next result provides the finite convergence property of Algorithm 1.

TheOREM 2. Let $T$ satisfy either $\mathbf{T 1}$ or $\mathbf{T} 2$. If $T$ satisfies $\mathbf{T 2}$, assume also that (2.5) holds true. Then Algorithm 1 converges to an exact solution of problem (1.2) in at most $n(n+1) / 2$ (inner) steps.

Proof Recall first that $Q^{0}=O$ and $\mathbf{x}^{k+1, \nu} \geq \mathbf{x}^{k}$ for all $k, \nu \geq 1$ (see (2.9)). Thus $\overline{P^{k+1, \nu}} \geq Q^{k}$. Moreover, within each external cycle, the inner iterates $\left\{\mathbf{x}^{k, \nu}\right\}$ are strictly decreasing (equality is excluded, because of the stopping criterion described in Lemma 3). Consider, in fact, two subsequent inner iterates, namely (2.3) and

$$
P^{k, \nu}\left(\mathbf{x}^{k, \nu+1}-\boldsymbol{l}\right)-Q^{k-1}\left(\mathbf{x}^{k, \nu+1}-\boldsymbol{u}\right)+T \mathbf{x}^{k, \nu+1}=\mathbf{b}-\boldsymbol{l} .
$$

By equating the left-hand sides of (2.3) and (2.10), one obtains

$$
\left(P^{k, \nu}-Q^{k-1}+T\right) \mathbf{x}^{k, \nu+1}=\left(P^{k, \nu}-Q^{k-1}+T\right) \mathbf{x}^{k, \nu}+\zeta^{k, \nu}
$$

where $\boldsymbol{\zeta}^{k, \nu}=-\left(P^{k, \nu}-P^{k, \nu-1}\right)\left(\mathbf{x}^{k, \nu}-\boldsymbol{l}\right) \leq \mathbf{0}$. Thus, since $P^{k, \nu}-Q^{k-1}+T$ is Stieltjes, one has $\left(P^{k, \nu}-Q^{k-1}+T\right)^{-1}>O$ and, hence, $\left\{\mathbf{x}^{k, \nu}\right\}$ is strictly decreasing. Consequently, $\left\{P^{k, \nu}\right\}$ is decreasing as well and, because $P^{0, \nu}=I$ and $P^{k, \nu} \geq Q^{k}$, by denoting $\left\{q_{i}^{k}\right\}$ the diagonal entries of $Q^{k}$, and setting

$$
m_{k}=\sum_{i=1}^{n} q_{i}^{k} \geq k
$$

each inner cycle will converge in at most $n-m_{k}$ steps.

Next, inequality (2.9) implies that the external iterates $\left\{\mathbf{x}^{k}\right\}$ generated by $(2.1)$ are not decreasing, i.e., 


$$
\mathbf{x}^{k+1} \geq \mathbf{x}^{k}, \quad k \geq 1
$$

Consequently, the sequence $\left\{Q_{k}\right\}$ is not decreasing with $Q^{0}=O$, and $Q^{k} \leq I$ for all $k$. Then, because of (2.11) and of the stopping creterion provided by Lemma 2 . It follows that the outer cycle will converge in $K \leq n$ cycles. Therefore, in conclusion, the total number of inner iterations is bounded by

$$
\sum_{k=1}^{K}\left(n-m_{k}\right) \leq \sum_{k=1}^{n}(n-k)=\frac{n(n+1)}{2} . \mathrm{u}
$$

REMARK 1. In practice, the determination of $\mathbf{x}^{k, \nu}$ from (2.3) can be accomplished quite efficiently by using a preconditioned conjugate gradient method (see, e.g., [9, 15]). This is particularly the case in applications where $T$ is a sparse and very large matrix (see Section 4). To this purpose, in light of the convergence property proved in Theorem 2, $\mathbf{x}^{k, \nu-1}$ (if $\nu>1$ ) or $\mathbf{x}^{k-1}($ if $\nu=1$ ) is conveniently used as a starting point for the conjugate gradient method (the effectiveness of this choice has been confirmed by a huge number of numerical tests).

REMARK 2. Even though Theorem 2 provides a finite convergence property for the inner-outer iteration (2.1)-(2.3), the resulting upper bound is rather large when the dimension $n$ of the problem is large. In practice, however, convergence is obtained in just a few iterations, as confirmed by several numerical tests.

COROLlary 2. If T satisfies T2, then (2.5) is a necessary and sufficient condition for problem (1.2) to have a solution.

Proof It has been already proved that (2.5) is a sufficient condition for problem (1.2) to have a solution. In order to prove that this condition is also necessary, from (1.2) one has

$$
\mathbf{v}^{T}(\mathbf{b}-\boldsymbol{l})=\mathbf{v}^{T}[P(\mathbf{x})(\mathbf{x}-\boldsymbol{l})+Q(\mathbf{x})(\boldsymbol{u}-\mathbf{x})]
$$

Moreover, from (1.3) and Lemma 1,

$$
\mathbf{0} \leq P(\mathbf{x})(\mathbf{x}-\boldsymbol{l})+Q(\mathbf{x})(\boldsymbol{u}-\mathbf{x}) \leq \boldsymbol{u}-\boldsymbol{l} .
$$

The two inequalities in (2.5) then follow.

2.1. A dual algorithm. For the sake of completeness, a corresponding dual Algorithm 2, with respect to Algorithm 1, is also described, where $Q^{k, \nu}=Q\left(\mathbf{x}^{k, \nu}\right)$, for all $k, \nu \geq 1$. Such an algorithm enjoys the same properties of Algorithm 1, which can be proved by means of similar arguments.

Observe that, when the solution $\mathbf{x} \leq \boldsymbol{u}$, then $Q^{0}=Q^{1}=Q(\mathbf{x})=O$ because of (2.12) and, consequently, Algorithm 1 requires only one outer iteration [3]. For the same reason, each outer iteration in Algorithm 2 requires only one inner iteration. Symmetrically, when the solution $\mathbf{x} \geq \boldsymbol{l}$, then $P^{0}=P^{1}=P(\mathbf{x})=I$ and, consequently, Algorithm 2 converges in one outer iteration. For the same reason, each outer iteration in Algorithm 1 requires only one inner iteration. 


\section{Algorithm 2}

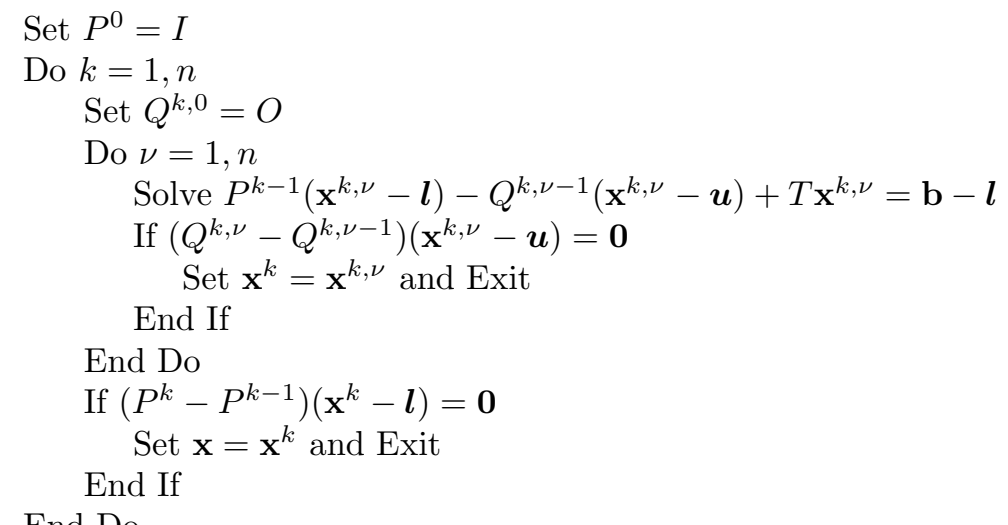

End Do

3. Uniqueness of the solution. In this section the conditions for the uniqueness of the solution of problem (1.2) are studied. To begin with, the simpler case in which $T$ satisfies $\mathbf{T} \mathbf{1}$ is analysed.

THEOREM 3. If $T$ satisfies $\mathbf{T 1}$, then the solution of problem (1.2) exists and is unique.

Proof The existence of a solution $\mathbf{x}$ of (1.2) has been already proved costructively by means of Algorithm 1. To prove its uniqueness, let $\mathbf{y}$ be another solution of the same problem. Consequently,

$$
P(\mathbf{x})(\mathbf{x}-\boldsymbol{l})-Q(\mathbf{x})(\mathbf{x}-\boldsymbol{u})+T \mathbf{x}=P(\mathbf{y})(\mathbf{y}-\boldsymbol{l})-Q(\mathbf{y})(\mathbf{y}-\boldsymbol{u})+T \mathbf{y} .
$$

Moreover, one has

$$
[P(\mathbf{y})(\mathbf{y}-\boldsymbol{l})-Q(\mathbf{y})(\mathbf{y}-\boldsymbol{u})]-[P(\mathbf{x})(\mathbf{x}-\boldsymbol{l})-Q(\mathbf{x})(\mathbf{x}-\boldsymbol{u})]=(\bar{P}-\bar{Q})(\mathbf{y}-\mathbf{x}),
$$

where $\bar{P}$ e $\bar{Q}$ are diagonal matrices, whose diagonal entries, $\left\{\bar{p}_{i}\right\}$ and $\left\{\bar{q}_{i}\right\}$, are respectively given by

$$
\bar{p}_{i}=\left\{\begin{array}{cll}
0 & \text { if } \quad x_{i}, y_{i}<l_{i}, \\
1 & \text { if } \quad x_{i}, y_{i} \geq l_{i}, \\
\frac{x_{i}-l_{i}}{x_{i}-y_{i}} & \text { if } \quad x_{i} \geq l_{i}>y_{i}, \\
\frac{y_{i}-l_{i}}{y_{i}-x_{i}} & \text { if } \quad y_{i} \geq l_{i}>x_{i},
\end{array} \quad \bar{q}_{i}=\left\{\begin{array}{cll}
0 & \text { if } \quad x_{i}, y_{i} \leq u_{i}, \\
1 & \text { if } \quad x_{i}, y_{i}>u_{i}, \\
\frac{x_{i}-u_{i}}{x_{i}-y_{i}} & \text { if } \quad x_{i}>u_{i} \geq y_{i}, \\
\frac{y_{i}-u_{i}}{y_{i}-x_{i}} & \text { if } \quad y_{i}>u_{i} \geq x_{i},
\end{array}\right.\right.
$$

so that

$$
\bar{P} \geq \bar{Q} \geq O
$$

From (3.1) and (3.2), one has

$$
(\bar{P}-\bar{Q}+T)(\mathbf{y}-\mathbf{x})=\mathbf{0} .
$$

Since $T$ satisfies T1, matrix $(\bar{P}-\bar{Q}+T)$ is Stieltjes, then nonsingular, and uniqueness $(\mathbf{y}=\mathbf{x})$ follows. 
In order to discuss the remaining case, the following definition is given.

Definition 1. Let $\mathbf{x}=\left(x_{i}\right)$ be a solution of (1.2). This is said to be an interior solution if

$$
\exists i \in\{1, \ldots, n\}: \quad l_{i}<x_{i}<u_{i} .
$$

THEOREM 4. If T satisfies T2 and (2.5) holds true, then problem (1.2) has at least one solution. Moreover,

1. if such a solution is an interior solution, then this is the only solution of the problem;

2. if more than one solution exist, then the difference of any two solutions belongs to the null space of matrix $T$.

Proof The existence of a solution $\mathbf{x}$ of (1.2) has been already proved costructively by means of Algorithm 1 (see also Corollary 2). Its uniqueness is now proved when it is an interior solution. Let $\mathbf{y}$ be another solution of the same problem. Consequently, (3.1) $-(3.5)$ hold true. Since $T$ satisfies T2, it suffices to show that $\bar{P} \neq \bar{Q}$. From (3.6) we know that there exists $i \in\{1, \ldots, n\}$ such that $l_{i}<x_{i}<u_{i}$. For such index $i$, one has

$$
\bar{p}_{i}-\bar{q}_{i}=\left\{\begin{array}{cl}
\frac{x_{i}-l_{i}}{x_{i}-y_{i}}>0, & \text { if } \quad y_{i}<l_{i}, \\
1, & \text { if } \quad l_{i} \leq y_{i} \leq u_{i}, \\
\frac{u_{i}-x_{i}}{y_{i}-x_{i}}>0, & \text { if } \quad y_{i}>u_{i},
\end{array}\right.
$$

so that $\bar{P} \neq \bar{Q}$. Therefore, matrix $\bar{P}-\bar{Q}+T$ is Stieltjes and uniqueness $(\mathbf{y}=\mathbf{x})$ follows from (3.5).

On the other hand, when $\mathbf{x}$ is not unique one has $\bar{P}=\bar{Q}$, so that (3.5) reduces to $T(\mathbf{y}-\mathbf{x})=\mathbf{0}$. That is, $\mathbf{y}-\mathbf{x} \in \operatorname{null}(T)$.

COROLlary 3. If T satisfies T2 and (2.5) holds true, then a non interior solution of problem (1.2) is generally not unique.

Proof Let $\omega_{u}$ and $\omega_{l}$ be the two sets of indices defined by

$$
\omega_{u}=\left\{i: x_{i} \geq u_{i}\right\}, \quad \omega_{l}=\left\{i: x_{i} \leq l_{i}\right\} .
$$

If $\mathbf{v}=\left(v_{i}\right)$, set

$$
\begin{gathered}
\alpha=\left\{\begin{array}{cc}
-\min _{i \in \omega_{u}}\left\{\left(x_{i}-u_{i}\right) / v_{i}\right\} & \text { if } \omega_{u} \neq \emptyset \\
-\infty & \text { otherwise }
\end{array}\right. \\
\beta=\left\{\begin{array}{cc}
\min _{i \in \omega_{l}}\left\{\left(l_{i}-x_{i}\right) / v_{i}\right\} & \text { if } \omega_{l} \neq \emptyset \\
\infty & \text { otherwise }
\end{array}\right.
\end{gathered}
$$

Clearly, $\alpha \leq 0 \leq \beta$. Moreover, one verifies that

$$
\mathbf{x}(\theta) \equiv \mathbf{x}+\theta \mathbf{v}, \quad \theta \in[\alpha, \beta],
$$

is a solution of the problem. 


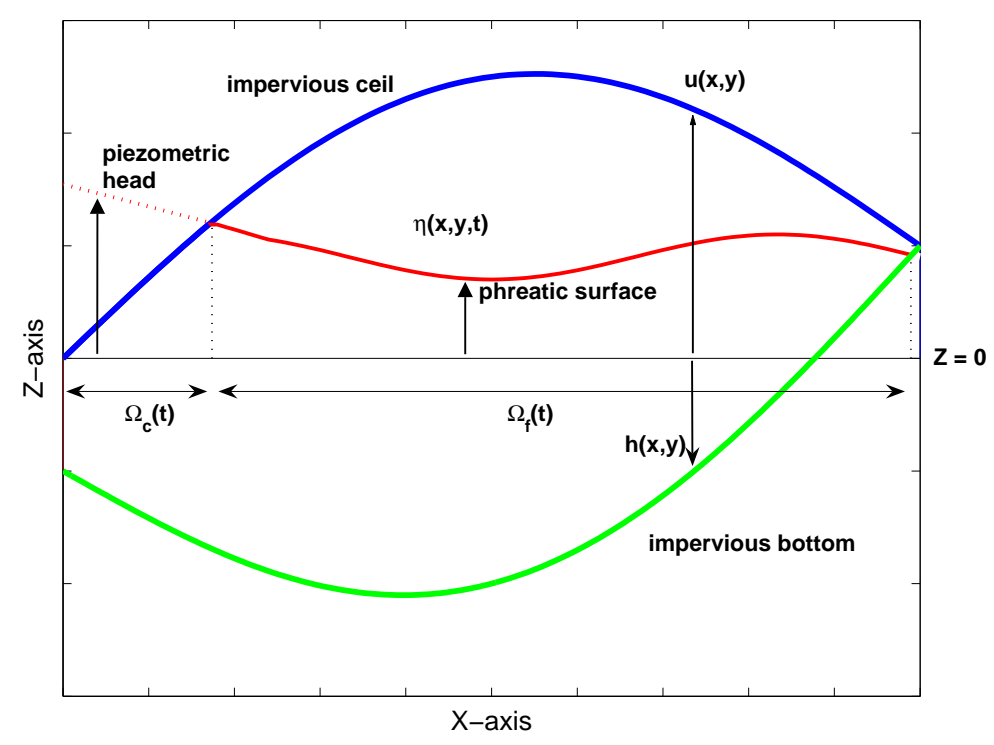

FIG. 4.1. Confined-unconfined aquifer.

The following example elucidates the previous arguments.

ExAmple 1. Let problem (1.2) be defined by (1.4). Consequently, T satisfies T2, with $\mathbf{v}=\boldsymbol{u}$, and (2.5) holds true. One verifies that $\mathbf{x}=(-1,2)^{T}$ is a (non interior) solution of (1.2). Moreover, according to Corollary 3, (3.7) is also a solution, with $\alpha=-1$ and $\beta=1$. Therefore, in such a case we have infinite (non interior) solutions to the problem. However, if we modify the right-hand side by setting $\mathbf{b}=(-1,2)^{T}$ we have that (2.5) holds true. Moreover, one verifies that a solution to the problem (1.2) is given by $\mathbf{x}=(0,1)^{T}$, which is a non interior solution. Nevertheless, according to Corollary 3, such a solution turns out to be unique, because in this case one obtains $\alpha=\beta=0$.

4. Modelling confined-unconfined flows in porous media. Consider the mathematical modelling of a two-dimensional flow in a homogeneous and isotropic aquifer. When a free-surface exists, the governing differential equations is given by the Boussinesq equation [1]

$$
\varepsilon \eta_{t}=\left[\kappa(h+\eta) \eta_{x}\right]_{x}+\left[\kappa(h+\eta) \eta_{y}\right]_{y}+\varphi,
$$

where (see Figure 4.1) $x$ and $y$ are coordinates in an horizontal reference frame; $t$ is the time; $\varepsilon$ and $\kappa$ are the porosity and the hydraulic conductivity, respectively (which are assumed to be constant); $h(x, y)$ is the prescribed aquifer's bottom and $\eta(x, y, t)$ is the (unknown) free-surface location; $\varphi(x, y, t)$ represents the prescribed source or sink and the time dependent domain is

$$
\Omega(t)=\{(x, y): h(x, y)+\eta(x, y, t)>0\}, \quad t>0 .
$$

Moreover, if the aquifer is confined from above by a ceil set at distance $u(x, y)$ from the vertical reference level, one has that (4.1) actually holds true only in the sub-domain

$$
\Omega_{f}(t)=\{(x, y) \in \Omega(t):-h(x, y)<\eta(x, y, t)<u(x, y)\}, \quad t>0,
$$


whereas, in

$$
\Omega_{c}(t)=\Omega(t) \backslash \bar{\Omega}_{f}(t), \quad t>0,
$$

the governing equation is

$$
0=\left[\kappa(h+\eta) \eta_{x}\right]_{x}+\left[\kappa(h+\eta) \eta_{y}\right]_{y}+\varphi .
$$

In such a case, the unknown $\eta(x, y, t)$ represents the piezometric head [1]. The two sub-domains (4.2) and (4.3) are obviously separated by the (unknown) line defined by

$$
\Gamma_{f c}(t)=\{(x, y): \eta=u\}
$$

The correct numerical solution of the mixed problem (4.1)-(4.4) is of interest in applications [6] and is non-trivial, because of the involvement of two moving boundaries [12]. For this purpose, consider a suitably large square containing $\Omega(t), t>0$, which is covered by a Cartesian grid having size $\Delta x$ and $\Delta y,\left(x_{i}, y_{j}\right)=(i \Delta x, j \Delta y)$. Moreover, for a given time step $\Delta t$, let $t_{\ell}=\ell \Delta t, \ell \geq 0$, denote the $\ell$-th time level. It is assumed the knowledge of $\varepsilon, \kappa, h_{i j}=h\left(x_{i}, y_{j}\right)$, and $u_{i j}=u\left(x_{i}, y_{j}\right)$, which are independent from time, and

$$
\varphi_{i j}^{\ell}=\varphi\left(x_{i}, y_{j}, t_{\ell}\right), \quad \ell \geq 0, \quad \eta_{i j}^{0}=\eta\left(x_{i}, y_{j}, 0\right) .
$$

Finally, the total water depth is defined by

$$
H_{i j}^{\ell}=\max \left\{0, \min \left\{h_{i j}+u_{i j}, h_{i j}+\eta_{i j}^{\ell}\right\}\right\},
$$

which yields $H_{i j}^{\ell}=0$ in the dry area, $H_{i j}^{\ell}=h_{i j}+\eta_{i j}^{\ell}$ in $\Omega_{f}(t)$, and $H_{i j}^{\ell}=h_{i j}+u_{i j}$ in $\Omega_{c}(t)$. Then, a finite difference discretization which is consistent with both Equations (4.1) and (4.4) is taken to be (see also [3, 4])

$$
\begin{aligned}
& \varepsilon\left(\frac{\max \left[-h_{i j}, \min \left(u_{i j}, \eta_{i j}^{\ell+1}\right)\right]-\max \left[-h_{i j}, \min \left(u_{i j}, \eta_{i j}^{\ell}\right)\right]}{\Delta t}\right) \\
& -\kappa\left(\frac{H_{i+\frac{1}{2}, j}^{\ell}\left(\eta_{i+1, j}^{\ell+1}-\eta_{i j}^{\ell+1}\right)-H_{i-\frac{1}{2}, j}^{\ell}\left(\eta_{i j}^{\ell+1}-\eta_{i-1, j}^{\ell+1}\right)}{\Delta x^{2}}\right. \\
& \left.+\frac{H_{i, j+\frac{1}{2}}^{\ell}\left(\eta_{i, j+1}^{\ell+1}-\eta_{i j}^{\ell+1}\right)-H_{i, j-\frac{1}{2}}^{\ell}\left(\eta_{i j}^{\ell+1}-\eta_{i, j-1}^{\ell+1}\right)}{\Delta y^{2}}\right)=\varphi_{i j}^{\ell} .
\end{aligned}
$$

Between grid points, the aquifer thicknesses $H_{i \pm 1 / 2, j}^{\ell}$ and $H_{i, j \pm 1 / 2}^{\ell}$ are defined as averages from the nearest grid values.

It is to be noted that for those grid points $(i, j)$ where $\varphi_{i j}^{\ell}=0, H_{i \pm 1 / 2, j}^{\ell}=0$, and $H_{i, j \pm 1 / 2}^{\ell}=0$, Equation (4.6) trivially implies (see (4.5)) $H_{i, j}^{\ell+1}=H_{i, j}^{\ell}=0$ (dry area). In this case (4.6) does not contribute to the system that is being formulated.

The remaining set of equations (corresponding to those grid points where at least one of $H_{i \pm 1 / 2, j}^{\ell}$ and $H_{i, j \pm 1 / 2}^{\ell}$ is strictly positive) can be assembled into a single piecewise linear system. Upon multiplication by $\Delta t / \varepsilon$ and by setting $l_{i j}=-h_{i j}$, 


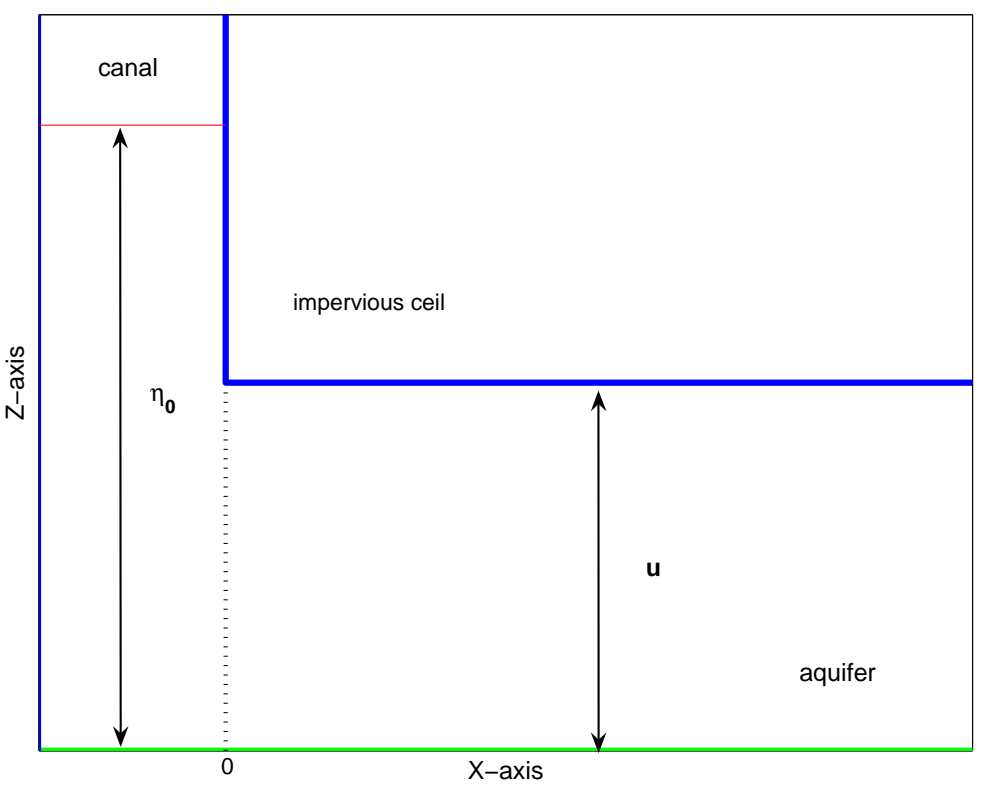

FIG. 4.2. Bounded recharging aquifer.

this system (which has to be solved at every time step) can be recognized of being in the form (1.1). The resulting matrix $T$ is irreducible, sparse, symmetric, positive semidefinite, and of time dependent size $n_{\ell} \times n_{\ell}$, with $n_{\ell}$ being the number of grid points $(i, j)$ where at least one of $H_{i \pm 1 / 2, j}^{\ell}$ and $H_{i, j \pm 1 / 2}^{\ell}$ is strictly positive. This matrix satisfies either $\mathbf{T} 1$, when the free-surface elevation is specified in at least one point of the boundary, or $\mathbf{T 2}$, when only the flow is specified at the boundary. According to Remark 1, a preconditioned conjugate gradient method is used for solving the resulting linear systems.

4.1. Bounded recharging aquifer. A first numerical test is presented, which is aimed to verify the accuracy of the discrete method (4.6). For this purpose, consider the simple case of a one dimensional confined-unconfined aquifer reported in Reference [12]. A canal (see Figure 4.2), confines with a bounded recharging aquifer whose ceil height is $u$. The aquifer is initially dry. Let $\eta_{0}$ be the (known) constant height of water in the canal. The following parameters are specified,

$$
\kappa=10^{-3} \pi \mathrm{m} / \mathrm{s}, \quad \varepsilon=10^{-1} \pi, \quad u=5 \mathrm{~m}, \quad \eta_{0}=5.5 \mathrm{~m},
$$

for which the analytical solution has been reported in [12, p. 154], Fig. 4.

A numerical simulation has been carried out by using a spatial stepsize $\Delta x=$ $2.4 \cdot 10^{-2} \mathrm{~m}$, and a time step $\Delta t=0.5 \mathrm{~s}$. Because of the prescribed level $\eta_{0}$ of water in the canal, matrix $T$ in the resulting piecewise linear system (1.2) is tridiagonal and satisfies T1. The simulation has been carried out for $T=900 \mathrm{~s}$. In almost all time steps, Algorithm 1 requires only 1 outer iteration, each requiring 1 inner iteration. The computed results shown in Figures 4.3 and 4.4 are in perfect agreement with the analytical solution reported in [12, p. 154], thus confirming the effectiveness of scheme (4.6). In particular, in Figure 4.3 the free-surface profiles are plotted in solid line, whereas the piezometric head profiles are plotted in dotted line, at different times. Figure 4.4 shows the amount of recharging water $Q(t)$. 
4.2. Free-surface dynamics in the proximity of a well. As a second test problem, consider the case in which the aquifer's bottom and ceil have been assumed to be symmetrical, with respect to the reference vertical level, both described by a paraboloid of revolution given by

$$
u(x, y) \equiv h(x, y)=h_{0}\left(1-\frac{x^{2}+y^{2}}{L^{2}}\right)
$$

where $h_{0}$ and $L$ are given positive constants. It is also assumed that at the initial time $t_{0}=0$ all the porous space is filled with water, so that $\eta(x, y, 0)=u(x, y)$ and, consequently, $\Omega(0)=\left\{(x, y): x^{2}+y^{2}<L^{2}\right\} \equiv \Omega_{c}(0)$. The resulting matrix $T$, which satisfies T2, is sparse, symmetric positive semidefinite, and of size $n^{\ell}$, which, at the initial time $t_{0}$, is $n^{\ell} \sim N^{2} \frac{\pi}{4}$.

A numerical simulation has been carried out for 14 days, by using $\Delta x=\Delta y=$ $2 L /(N+1)$ and a relatively large time step size $\Delta t=1 d a y$. For the present simulations the chosen parameters are $\varepsilon=0.4, \kappa=1 \mathrm{~m} / \mathrm{s}, h_{0}=10 \mathrm{~m}$ and $L=10^{3} \mathrm{~m}$. The flow is then driven by an idealized pointwise sink, representing a well located at the origin, that pumps water out of the aquifer at a constant rate $q=10 \mathrm{~m}^{3} / \mathrm{s}$. Thus, by setting $\varphi_{i j}^{\ell}=0$, except $\varphi_{00}^{\ell}=-\frac{q}{\Delta x \Delta y}$, the new water volume at time $t_{\ell+1}$ is given by

$$
V^{\ell+1}=\varepsilon \Delta x \Delta y \sum_{i j} H_{i j}^{\ell+1}=V^{\ell}-q \Delta t
$$

Figure 4.5 shows, in solid line, the resulting free-surface elevation (and, where appropriate, the piezometric head in dotted line) $\eta_{i 0}^{\ell}$ at the cross section $y=0$, for $\ell=0,1, \ldots, 14$.

For specified grid resolution corresponding to $N=50,100$, and 200, Table 4.1 shows, for each time step, the number of outer $\left(k_{\text {out }}\right)$ and total inner $\left(\nu_{t o t}\right)$ iterations required, along with the size $n^{\ell}$ of the resulting system and, finally, the computed water volume at each time step. As one expects from Figure $4.5, n^{\ell}$ is constant for the first 8 time levels and then decreases in the subsequent ones. Moreover, the number of both inner and outer iterations turns out to be remarkably small and practically insensitive to grid resolution.

Table 4.1 also shows that, for all $\ell=1,2, \ldots, 14$, the water volumes are strictly positive and linearly decreasing at a constant rate. In fact, the volume difference between two subsequent time levels (see Equation (4.8)) is correctly given by $V^{\ell+1}-$ $V^{\ell}=-q \Delta t=-864,000 \mathrm{~m}^{3}$. Thus, any attempt to extend the simulation beyond day 14 would produce a physically unrealistic negative water volume $V^{15}<0$. It can be shown that this implies that the right-hand side in Equation (4.6) violates the first inequality in (2.5). Consequently, when $\ell=15$ Corollary 2 applies indicating that problem (4.6) does not have a solution. This is an interesting example demonstrating that the proposed algorithm does not permit artificial over-drainage.

It is also worth noting that in the first 7 time levels, $\eta_{i j}$ is everywhere no less than $l_{i j}$. Consistently with that observed in Section 2.1, convergence is achieved in only one inner iteration per outer iteration. On the other hand, starting from time level $9, \eta_{i j}$ is everywhere no larger than $u_{i j}$ and only one outer iteration is required for convergence.

4.3. Evolution to steady state. As a third and final test problem, another aquifer is considered, with the same functions $h(x, y)$ and $u(x, y)$ as defined in (4.7), 
TABLE 4.1

Numerical results for the second test problem.

\begin{tabular}{|c|c|c|c|c|c|c|c|c|c|c|c|c|}
\hline & \multicolumn{4}{|c|}{$N=50$} & \multicolumn{4}{|c|}{$N=100$} & \multicolumn{4}{|c|}{$N=200$} \\
\hline $\bar{\ell}$ & $k_{\text {out }}$ & $\nu_{t o t}$ & $\overline{n_{\ell}}$ & $V_{\ell}$ & $k_{\text {out }}$ & $\nu_{t o t}$ & $n_{\ell}$ & $V_{\ell}$ & $k_{\text {out }}$ & $\nu_{t o t}$ & $n_{\ell}$ & $\overline{V_{\ell}}$ \\
\hline 0 & - & - & - & 12564992 & - & - & - & 12566221 & - & - & - & 12566346 \\
\hline 1 & 5 & 5 & 2085 & 11700992 & 5 & 5 & 8109 & 11702221 & 5 & 5 & 31965 & 11702346 \\
\hline 2 & 3 & 3 & 2085 & 10836992 & 4 & 4 & 8109 & 10838221 & 4 & 4 & 31965 & 10838346 \\
\hline 3 & 3 & 3 & 2085 & 9972992 & 3 & 3 & 8109 & 9974221 & 3 & 3 & 31965 & 9974346 \\
\hline 4 & 3 & 3 & 2085 & 9108992 & 3 & 3 & 8109 & 9110221 & 3 & 3 & 31965 & 9110346 \\
\hline 5 & 2 & 2 & 2085 & 8244992 & 3 & 3 & 8109 & 8246221 & 3 & 3 & 31965 & 8246346 \\
\hline 6 & 3 & 3 & 2085 & 7380992 & 3 & 3 & 8109 & 7382221 & 3 & 3 & 31965 & 7382346 \\
\hline 7 & 3 & 3 & 2085 & 6516992 & 3 & 3 & 8109 & 6518221 & 3 & 3 & 31965 & 6518346 \\
\hline 8 & 2 & 5 & 2085 & 5652992 & 2 & 5 & 8109 & 5654221 & 2 & 6 & 31965 & 5654346 \\
\hline 9 & 1 & 2 & 2025 & 4788992 & 1 & 3 & 7793 & 4790221 & 1 & 3 & 30597 & 4790346 \\
\hline 10 & 1 & 3 & 1877 & 3924992 & 1 & 3 & 7177 & 3926221 & 1 & 3 & 28177 & 3926346 \\
\hline 11 & 1 & 3 & 1693 & 3060992 & 1 & 3 & 6533 & 3062221 & 1 & 3 & 25621 & 3062346 \\
\hline 12 & 1 & 3 & 1509 & 2196992 & 1 & 3 & 5797 & 2198221 & 1 & 3 & 22689 & 2198346 \\
\hline 13 & 1 & 3 & 1297 & 1332992 & 1 & 3 & 4929 & 1334221 & 1 & 3 & 19349 & 1334346 \\
\hline 14 & 1 & 4 & 1033 & 468992 & 1 & 4 & 3909 & 470221 & 1 & 4 & 15249 & 470346 \\
\hline
\end{tabular}

TABLE 4.2

Numerical results for the third test problem.

\begin{tabular}{|r|rrr|rrr|rrr|}
\hline & \multicolumn{2}{|c|}{$N=50, V=6282496$} & \multicolumn{2}{|c|}{$N=100, V=6283110$} & \multicolumn{2}{c|}{$N=200, V=6283173$} \\
\hline$\ell$ & $k_{\text {out }}$ & $\nu_{\text {tot }}$ & $n_{\ell}$ & $k_{\text {out }}$ & $\nu_{\text {tot }}$ & $n_{\ell}$ & $k_{\text {out }}$ & $\nu_{\text {tot }}$ & $n_{\ell}$ \\
\hline 10 & 2 & 3 & 1889 & 2 & 4 & 7277 & 2 & 4 & 28577 \\
20 & 2 & 3 & 1926 & 2 & 3 & 7444 & 2 & 3 & 29247 \\
30 & 2 & 3 & 1957 & 3 & 4 & 7582 & 2 & 3 & 29802 \\
40 & 2 & 3 & 1989 & 2 & 3 & 7693 & 2 & 3 & 30228 \\
50 & 2 & 3 & 2006 & 2 & 3 & 7774 & 2 & 3 & 30564 \\
60 & 1 & 2 & 2022 & 2 & 3 & 7828 & 2 & 3 & 30823 \\
70 & 2 & 2 & 2036 & 2 & 3 & 7889 & 2 & 3 & 31026 \\
80 & 1 & 1 & 2049 & 2 & 3 & 7923 & 2 & 3 & 31205 \\
90 & 2 & 2 & 2057 & 2 & 3 & 7972 & 2 & 3 & 31340 \\
100 & 1 & 1 & 2063 & 1 & 2 & 7994 & 2 & 3 & 31459 \\
110 & 2 & 2 & 2065 & 2 & 3 & 8010 & 2 & 3 & 31545 \\
120 & 1 & 1 & 2069 & 1 & 2 & 8029 & 2 & 3 & 31614 \\
130 & 1 & 1 & 2073 & 2 & 3 & 8041 & 2 & 3 & 31670 \\
140 & 1 & 1 & 2079 & 1 & 1 & 8067 & 2 & 2 & 31738 \\
150 & 1 & 1 & 2083 & 1 & 1 & 8075 & 2 & 3 & 31768 \\
\hline
\end{tabular}

and the initial water level and piezometric head $\eta(x, y, 0)$ being flat, at a slope, as indicated in Figure 4.6.

A numerical simulation has been carried out for 15 hours, by using a time step size $\Delta t=0.1$ hour. The chosen parameters are the same as in the previous test problem, and with $\varphi_{i j}^{\ell} \equiv 0$.

For specified $N=50,100$, and 200, Table 4.2 shows, at selected time steps, the number of outer $\left(k_{\text {out }}\right)$ and total inner $\left(\nu_{\text {tot }}\right)$ iterations required for convergence, along with the size $n^{\ell}$ of the resulting system. As expected, during the whole simulation the water volume is exactly conserved. Moreover, as shown in Figure 4.6, as time advances, the phreatic surface approaches its steady state configuration. Also in this 
case, the number of both inner and outer iterations is remarkably small and almost insensitive to grid resolution, thus confirming the usefulness of the proposed algorithm for real world applications.

5. Conclusions. Two simple algorithms based on a nested iterative procedure, aimed to solve certain piecewise linear systems that arise from the numerical modelling of free-surface hydrodynamics, have been described and analysed.

It is shown that, under rather general assumptions, the iterates are well defined and converge to the exact solution in a finite number of steps. Existence of the solution has been established under the same assumptions for which convergence is assured. Moreover, convergence to a solution is guaranteed also in the case where there is no uniqueness. In such a case, it has been shown how to retrieve all solutions of the problem.

Simple, and yet non-trivial, numerical tests have confirmed the efficiency, the robustness, and the usefulness of the proposed algorithms for real world applications to flows in confined, unconfined and mixed confined-unconfined aquifers.

\section{REFERENCES}

[1] J. Bear, A. Verruijt, Modeling Groundwater Flow and Pollution, D. Reidel Publ. Co., Dordrecht, Holland, 1987.

[2] S. Bellavia, M. Macconi, B. Morini, An affine scaling trust-region approach to boundconstrained nonlinear systems, Appl. Numer. Math. 44 (2003) 257-280.

[3] L. Brugnano, V. Casulli, Iterative solution of piecewise linear systems, SIAM Jour. on Scientific Computing, 30 (2008) 463-472.

[4] V. Casulli, Semi-implicit finite difference methods for the two-dimensional shallow water equations, Jour. of Computational Physics, 86 (1990) 56-74.

[5] V. Casulli, A high resolution wetting and drying algorithm for free-surface hydrodynamics, Intern. Jour. for Numer. Meth. in Fluids (2008) in press (DOI: 10.1002/fld.1896)

[6] C.X. Chen, L.T.Hu, X.S. WAng, Analysis of steady ground water flow toward wells in a confined-unconfined aquifer, Ground Water 44 (2006) 609-612.

[7] C.W. Cryer, Successive overrelaxation methods for solving linear complementarity problems arising from free boundary value problems, Free boundary value problems, Vol. I (Pavia, 1979) 109-131, INdAM "F. Severi", Rome, 1980.

[8] C.W. Cryer, The efficient solution of linear complementarity problems for tridiagonal Minkowski matrices, ACM TOMS 9 (1983) 199-214.

[9] G. H. Golub and C. F. van Loan, Matrix Computations, 3rd ed., The Johns Hopkins University Press, Baltimore, MD, 1996.

[10] R. A. Horn, C. R. Johnson, Topics in Matrix Analysis, Cambridge University Press, New York, 1991.

[11] C. Kanzow, A. KLug, An interior-point affine-scaling trust-region method for semismooth equations with box constraints, Comput. Optim. Appl. 37 (2007) 329-353.

[12] L. Li, D.A. Lockington, D.A. Barry, J.-Y. Parlange, P. Perrochet, Confined-unconfined flow in a horizontal aquifer, Jour. of Hydrology, 271 (2003) 150-155.

[13] Y. LiN, C.W. CRYER, An alternating direction implicit algorithm for the solution of linear complementarity problems arising from free boundary value problems, Appl. Math. Optim. 13 (1985) 1-17.

[14] J.-S. PANG, L. QI, Nonsmooth equations: motivations and algorithms, SIAM Jour. on Optimization 3 (1993) 443-465.

[15] Y.SAAD, Iterative Methods for Sparse Linear Systems, Second Edition, SIAM, Philadelphia, 2003. 


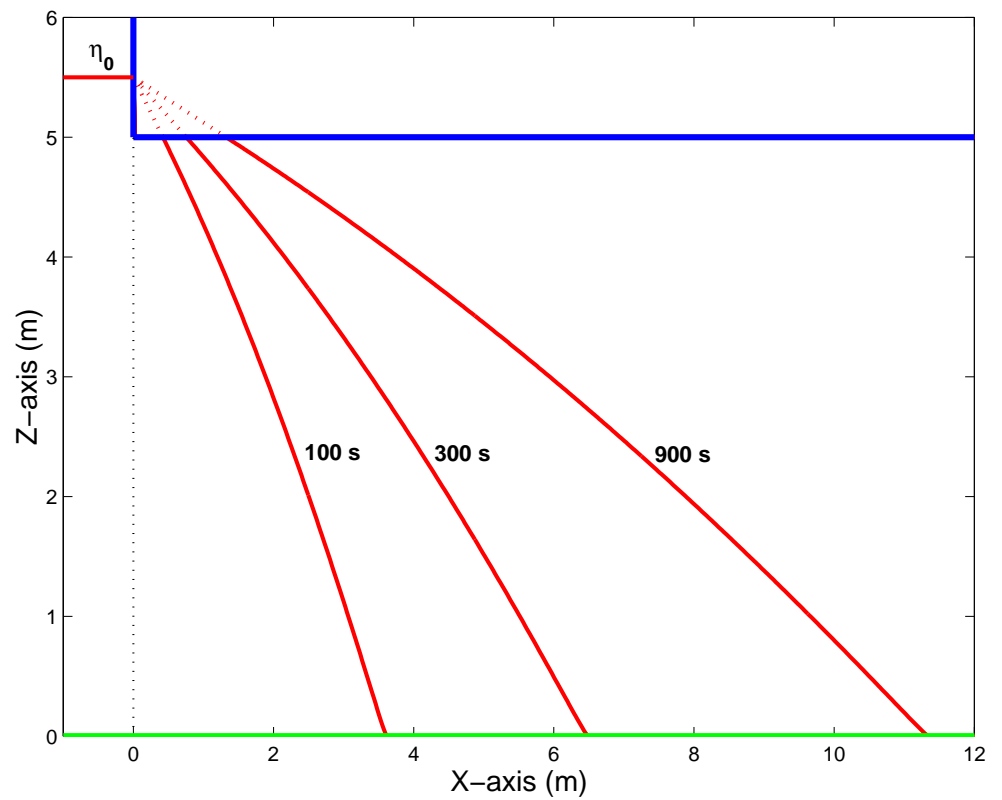

FIG. 4.3. Predicted free-surface and piezometric head profiles at different times.

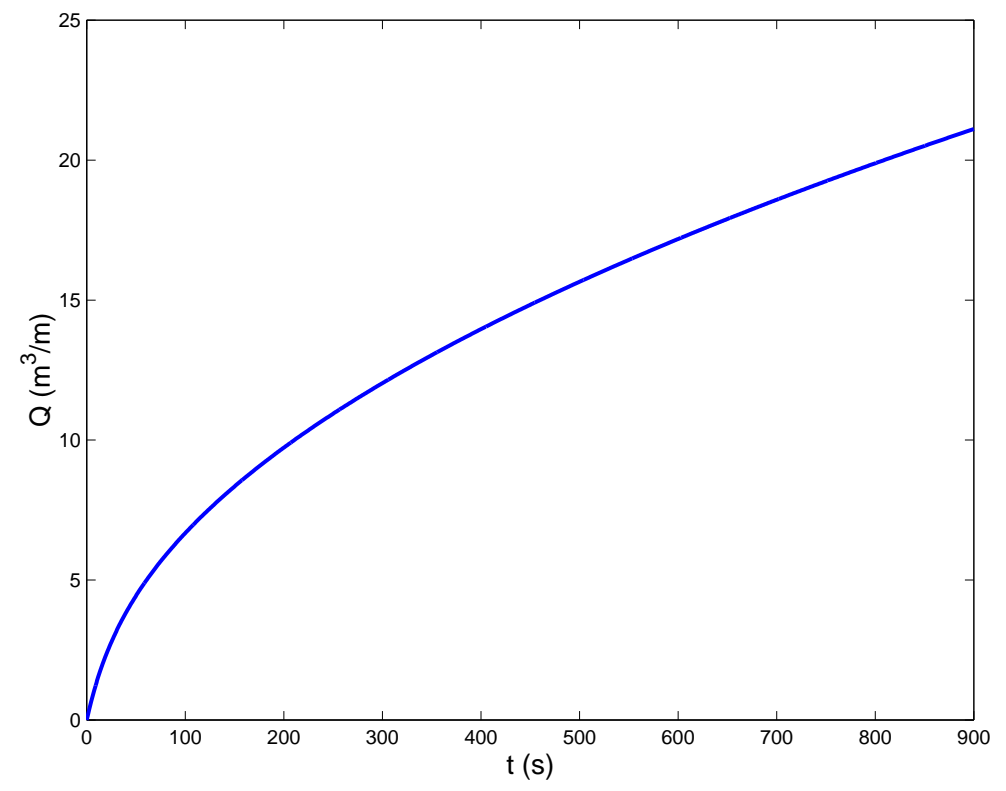

FIG. 4.4. Amount of recharging water. 


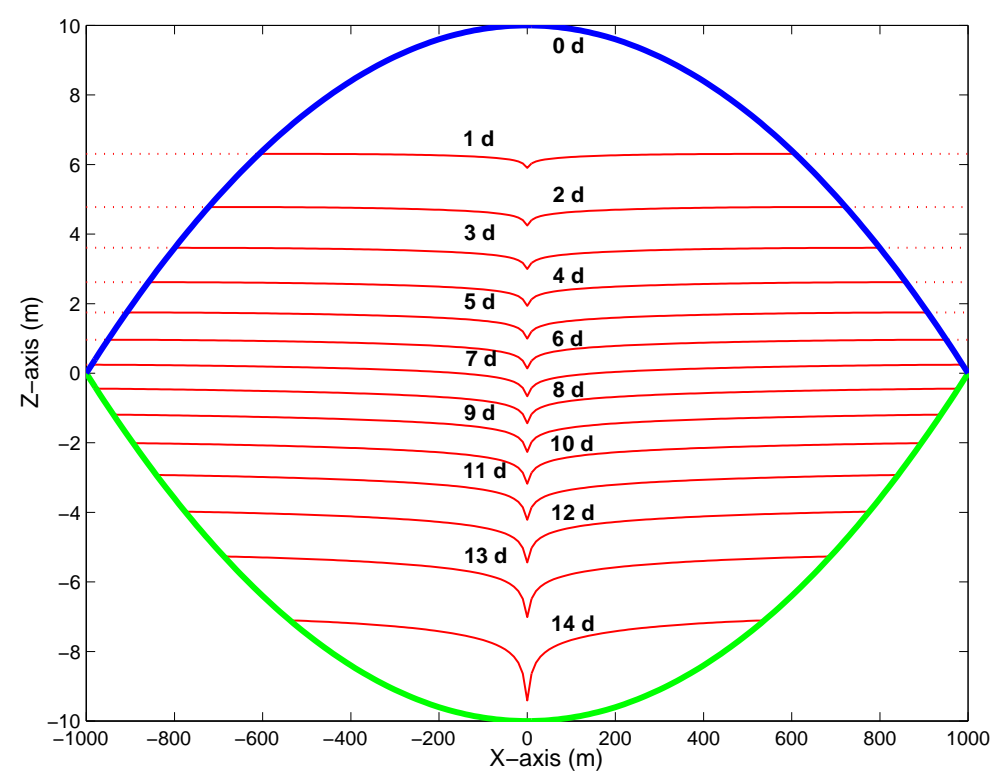

FIG. 4.5. Computed free surfaces at the cross section $y=0$.

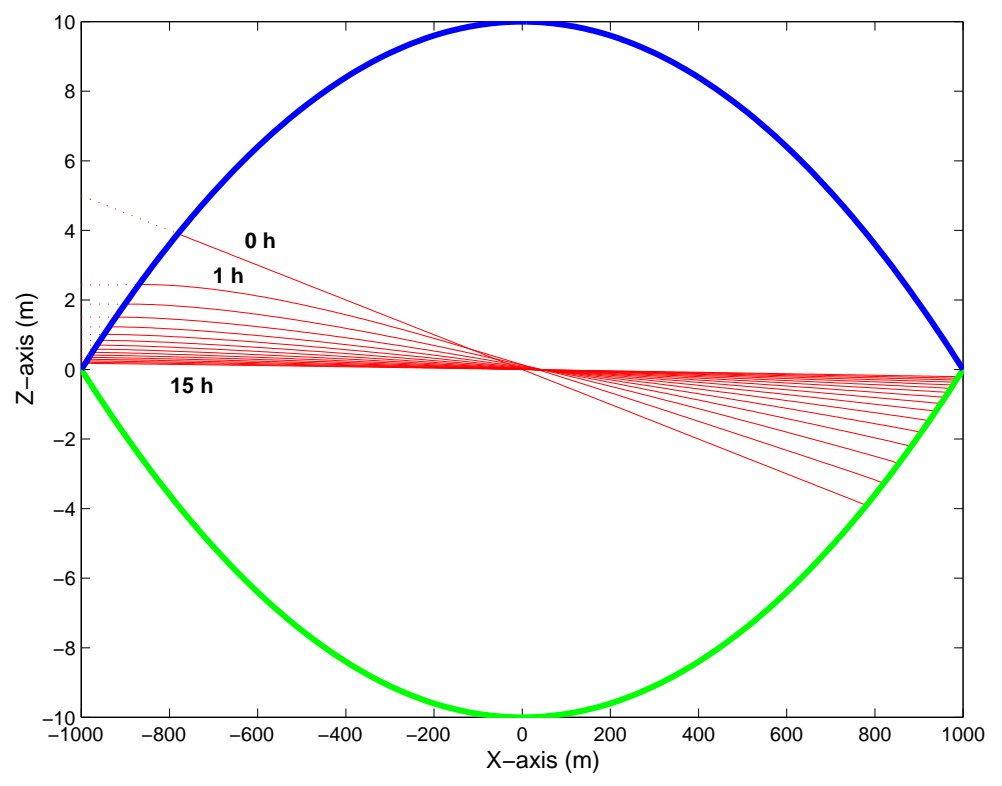

FIG. 4.6. Time evolution to steady state at the cross section $y=0$. 